\title{
Total presentation time and trials to criterion in connected discourse learning
}

\author{
DAVID J. KING \\ State University College, Oswego, New York 13126
}

\begin{abstract}
Seven sets of data are presented which summarize the relationship between trials to criterion and total presentation time for a 25-word-long passage of connected discourse. The resultant relationship shows a sharp negative deceleration with trials to criterion becoming quickly asymptotic between two and two and one-half trials. Implications of this relationship to the total-time hypothesis for connected discourse learning are briefly presented.
\end{abstract}

A number of previous studies (King, 1971, 1972, 1973a, b, 1974) have evaluated the total-time hypothesis for connected discourse learning. Most of these studies have focused, appropriately enough, on the dependent variable of total learning time as the best test of the total-time hypothesis. Concurrently, throughout all of these studies, information on trials to criterion has also been accumulating. The purpose of this note is to report, in summary form, on the relationship between total presentation time and trials to criterion as contained in seven sets of data taken from the above cited five studies.

This section presents a number of overall procedural questions. No pretense is made of presenting the details of each study. This information is available in the original publications. While considerable liberty has been taken below in combining a number of different conditions, the learning material had to be held constant. All of the information that follows concerns the relationship between total presentation time and number of trials required to reach a criterion of one perfect recall for a 25 -word-long passage. The specific passage was: "The early morning sun shining through the yellow curtain gave the bedroom a translucent quality. George opened one eye and quickly closed it in disgust." All work reported here also used a discrete one-word-at-a-time type of presentation. The modes of presentation used to present the learning material were both auditory (King, 1973b) and visual (all other references). In those studies using visual presentation, some used a carousel projector (King, 1971, 1972, Experiment I), some a motion analysis projector with 16-mm film (King, 1973a, b, 1974), and, in one case, both methods of stimulus presentation (King, 1972, Experiment II). The concept of total presentation time is a complex one and this is not the appropriate place for a detailed analysis. In the present context, suffice it to say that total presentation time is an overall value composed, from study to study, of various combinations of exposure interval, interitem interval, and rate of presentation. In all cases, the instructions to the learner were the same. That is, each $\mathrm{S}$ was asked to view (or listen to) the learning material and, following presentation, to recall the material by writing it down in a column (one word on each line) as close to the original as possible. This alternation of presentation of the learning material followed by a recall was continued until the $\mathrm{S}$ produced a perfect recall. Only a handfull of Ss were eliminated for failure to learn in a reasonable number of trials. On course, no $\mathrm{S}$ was used under more than one condition. The data presented below are based on an overall $\mathrm{N}$ of $500 \mathrm{Ss}$, equally divided between males and females. The $\mathrm{N}$ for each set of data may be determined by referral to the original experiments.

Figure 1 presents a summary of the relationship between total presentation time and trials to criterion for the above-described seven sets of data. Despite the many different conditions and procedures as enumerated above, a reasonably clear and consistent pattern emerges. With very brief total presentation times $(5 \mathrm{sec}$ is almost approaching a perceptual limit for the presentation of a 25-word-long passage on a discrete basis), a large number of trials are necessary for complete learning. The number of trials required becomes rather quickly asymptotic at between two and two and one-half trials as total presentation time increases. Apparently, within the limits of this specifis subject material and length of meterial, the rehearsal processes that occur with sufficiently long presentation times are maximally effective in reducing the number of trials required for complete learning to not less than two. Changing the complexity and/or length of the learning material would, of course, alter the specifics of the curve. On the other hand, the general nature of the curve is assumed to hold (i.e., for reasonably long passages, an asymptotic approach above one trial).

The general implications of the above conclusions for the total-time hypothesis for connected discourse learning is fairly clear. With total presentation time increasing and trials to criterion reasonably constant, the total-time hypothesis cannot hold at longer presentation times. This, coupled with the finding of a longer total learning time at very brief total presentation times (King, 1974) suggests an overall U-shaped relationship 


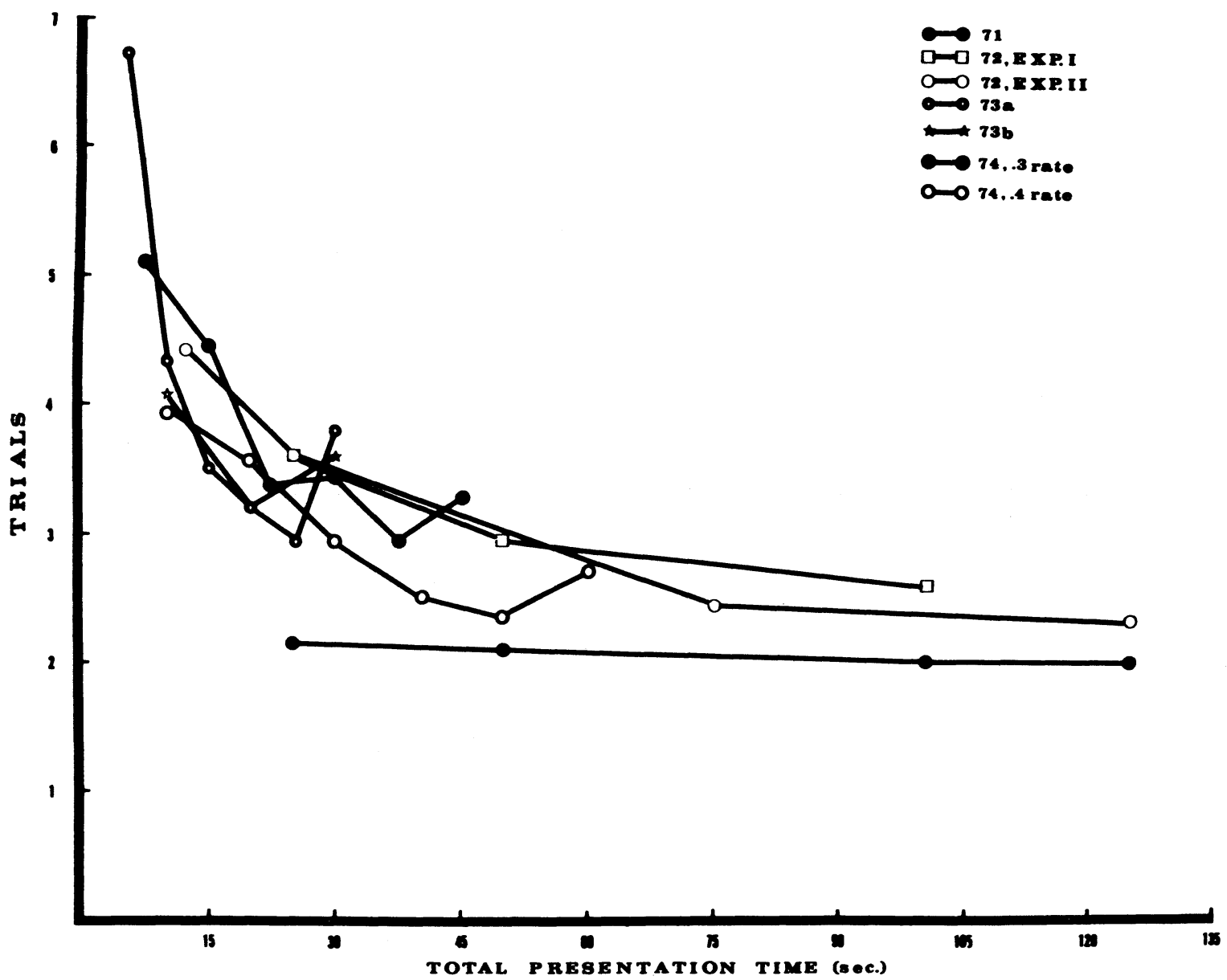

Fig. 1. Trials to criterion as a function of total presentation time for seven sets of data.

between total learning time and total presentation time.

\section{REFERENCES}

King, D. J. Influence of interitem interval in the learning of connected discourse. Journal of Experimental Psychology, $1971,87,132-134$.

King, D. J. Temporal factors involved in the learning of connected discourse. The Journal of General Psychology, $1972,87,187-194$.

King, D. J. Influence of exposure interval and interitem interval on the learning of connected discourse. Journal of
Experimental Psychology, 1973a, 97, 258-260.

King, D. J. Presentation time and method of reading in the learning of connected discourse. The Journal of General Psychology, 1973b, 88, 283-289.

King, D. J. Total presentation time and total learning time in connected discourse learning. Journal of Experimental Psychology, 1974, in press.

(Received for publication March 13, 1974.) 